\title{
Article
}

Subscriber access provided by Gothenburg University Library

\section{Dielectric Meta-Holograms Enabled with Dual Magnetic Resonances in Visible Light}

Zile Li, Inki Kim, Lei Zhang, Muhammad Q Mehmood, Muhammad S Anwar, Murtaza Saleem, Dasol Lee, Ki Tae Nam, Shuang Zhang, Boris S. Luk`yanchuk, Yu Wang, Guoxing Zheng, Junsuk Rho, and Cheng-Wei Qiu ACS Nano, Just Accepted Manuscript • DOI: 10.1021/acsnano.7b04868 • Publication Date (Web): 12 Sep 2017

Downloaded from http://pubs.acs.org on September 13, 2017

\section{Just Accepted}

"Just Accepted" manuscripts have been peer-reviewed and accepted for publication. They are posted online prior to technical editing, formatting for publication and author proofing. The American Chemical Society provides "Just Accepted" as a free service to the research community to expedite the dissemination of scientific material as soon as possible after acceptance. "Just Accepted" manuscripts appear in full in PDF format accompanied by an HTML abstract. "Just Accepted" manuscripts have been fully peer reviewed, but should not be considered the official version of record. They are accessible to all readers and citable by the Digital Object Identifier (DOI®). "Just Accepted" is an optional service offered to authors. Therefore, the "Just Accepted" Web site may not include all articles that will be published in the journal. After a manuscript is technically edited and formatted, it will be removed from the "Just Accepted" Web site and published as an ASAP article. Note that technical editing may introduce minor changes to the manuscript text and/or graphics which could affect content, and all legal disclaimers and ethical guidelines that apply to the journal pertain. ACS cannot be held responsible for errors or consequences arising from the use of information contained in these "Just Accepted" manuscripts. 


\section{Dielectric Meta-Holograms Enabled with Dual Magnetic Resonances in Visible Light}

Zile Li, ${ }^{\hbar, \curvearrowright}$ Inki Kim, ${ }^{\oint, \Omega}$ Lei Zhang, ${ }^{\dagger, \Omega}$ Muhammad Q. Mehmood, ${ }^{\#, \Omega}$ Muhammad S. Anwar, ${ }^{\&}$ Murtaza Saleem, ${ }^{\&}$ Dasol Lee, ${ }^{\S}$ Ki Tae Nam, ${ }^{\nabla}$ Shuang Zhang, ${ }^{\perp}$ Boris Luk'yanchuk, ${ }^{\ddagger}$ Yu Wang, ${ }^{,}$ Guoxing Zheng, ${ }^{\ddagger, \$, *}$ Junsuk Rho, ${ }^{\S, \bullet} *$ and Cheng-Wei Qiu ${ }^{\prime,=}, *$

School of Electronic Information, Wuhan University, Wuhan 430072, China

${ }^{s}$ Department of Mechanical Engineering, Pohang University of Science and Technology (POSTECH), Pohang 37673, Republic of Korea

Key Laboratory for Physical Electronics and Devices of the Ministry of Education \& Shaanxi Key Lab of Information Photonic Technique, Xi' an Jiaotong University, Xi'an 710049, China

\#Department of Electrical Engineering, Information Technology University of the Punjab, Ferozpur Road, 54000 Lahore, Pakistan

${ }^{\&}$ Department of Physics, Syed Babar Ali School of Science and Engineering (SBASSE), Lahore University of Management Sciences (LUMS), Opposite Sector U, D.H.A. Lahore 54792, Pakistan

${ }^{\top}$ Department of Materials Science and Engineering, Seoul National University, Seoul 08826, Republic of Korea

${ }^{\perp}$ School of Physics \& Astronomy, University of Birmingham, Birmingham B15 2TT, UK 


\author{
${ }^{£}$ Data Storage Institute, A*STAR (Agency for Science, Technology and Research), 2 \\ Fusionopolis Way, \#08-01, Innovis 138634, Singapore \\ ${ }^{\$}$ State Key Laboratory of Optical Communication Technologies and Networks, Wuhan Research \\ Institute of Posts \& Telecommunications, Wuhan 430074, China \\ •Department of Chemical Engineering, Pohang University of Science and Technology \\ (POSTECH), Pohang 37673, Republic of Korea \\ 'Department of Electrical and Computer Engineering, National University of Singapore, 4 \\ Engineering Drive 3, Singapore, 117583, Singapore \\ "SZU-NUS Collaborative Innovation Center for Optoelectronic Science and Technology, \\ Shenzhen University, Shenzhen 518060, China
}

\begin{abstract}
Efficient transmission-type meta-holograms have been demonstrated using highindex dielectric nanostructures based on Huygens' principle. It is crucial that the geometry size of building blocks needs to be judiciously optimized individually for spectral overlap of electric and magnetic dipoles. In contrast, reflection-type meta-holograms using the metal/insulator/metal scheme and geometric phase can be readily achieved with high efficiency and small thickness. Here, we demonstrate a general platform for design of dual magnetic resonance-based meta-holograms based on the geometric phase using silicon nanostructures that are quarter wavelength thick for visible light. Significantly, the projected holographic image can be unambiguously observed without a receiving screen even under the illumination of natural light. With the facilitation of the well-developed semiconductor industry, our ultra-thin magnetic resonance-based meta-holograms may have promising applications in anti-counterfeiting and information security.
\end{abstract}


KEYWORDS: metasurfaces, dielectric nanostructures, magnetic resonance, image hologram

Metasurfaces have led to many unconventional optical properties as well as novel physical phenomena and applications. ${ }^{1-32}$ In particular, geometric metasurfaces (GEMSs) based on the Pancharatnam-Berry phase have attracted extensive attention due to their simple and robust phase control scheme. ${ }^{16-32}$ The beauty of this approach lies in the linear dependence of phase delay $\varphi$ on the orientation angle $\alpha$ of each nanostructure, i.e., $\varphi= \pm 2 \alpha$, with the sign determined by the polarity of incident light. ${ }^{22,31}$ At the same time, the scattering amplitude remains unchanged since the geometry remains consistent. Therefore, GEMSs offer new perspectives in designing complex phase-only optical elements, such as holograms, ${ }^{25-34}$ which have been investigated in the visible and infrared ranges using noble metal nanostructures. ${ }^{33,34}$ For example, a reflectiontype hologram based on the hybrid of geometric phase and gap plasmon has been realized with high efficiency using metal-insulator-metal (MIM) structures. ${ }^{31,32}$ However, the intrinsic ohmic loss of metals is still severe at short visible frequency, which may deteriorate the total efficiency. Therefore, low-loss or even lossless dielectrics represent a promising material route towards high performance GEMSs. ${ }^{20-26}$ Nevertheless, dielectric GEMSs usually suffer from requirements of larger structural heights, in sharp contrast to metal GEMSs. For instance, the height of current dielectric structures is $\sim \lambda$ for $\mathrm{TiO}_{2}{ }^{23,24}$ and $\sim \lambda / 1.7$ for silicon, ${ }^{22}$ while that of metal structures can be as small as $\sim \lambda / 27 .{ }^{31}$ Consequently, high aspect ratio (AR), defined as the ratio of height to width, for instance, $6.3 \sim 15$ for $\mathrm{TiO}_{2}^{23,24}$ in the visible range and 4.8 for silicon ${ }^{22}$ in fiber telecommunication windows, is unavoidable for building blocks of GEMSs. The challenge in high AR seriously increases the fabrication burden and renders the device less compact. 
Although atomic layer deposition (ALD) enables fabrication of high AR dielectric nanostructures, ${ }^{24}$ it is still challenging to realize large-scale and cost-efficient samples.

Even though the generation of geometric phase is independent of the specific size, introduction of a resonant mode is also beneficial for optimized efficiency at a given wavelength. Lately, optical magnetic modes have been observed in high index insulators in the visible frequency range. ${ }^{35-41}$ In particular, by matching the electric dipole mode and magnetic dipole in frequency domain, Huygens' metasurfaces were demonstrated to manipulate transmitted light with high efficiency. $^{42,43}$ According to Mie theory, at given wavelength, the structure supporting a magnetic dipole mode has an even smaller size in comparison with the counterpart supporting an electric dipole mode. As a result, magnetic resonance is useful for shrinking the AR of structures for compact GEMSs design. Moreover, the scattering cross section of magnetic dipole mode is also superior to the electric counterpart, a higher manipulation efficiency is thus expected.

In this work, we demonstrate a silicon ( $\mathrm{Si}$ ) GEMS by combining geometric phase and magnetic resonance. The height of building blocks is dramatically reduced down to a quarter of the operation wavelength, while all advantages of conventional GEMSs are preserved. Three examples are presented to verify our proposal, including a Fourier hologram and two image holograms. For image holograms, clear holographic images can be observed under arbitrary illumination condition such as natural light, which greatly facilitate practical applications under loose requirements of illumination. In addition, superior image hologram performance is also demonstrated by Si GEMSs fabricated on silicon on insulator (SOI), which indicates a good compatibility with the mature semiconductor industry. 

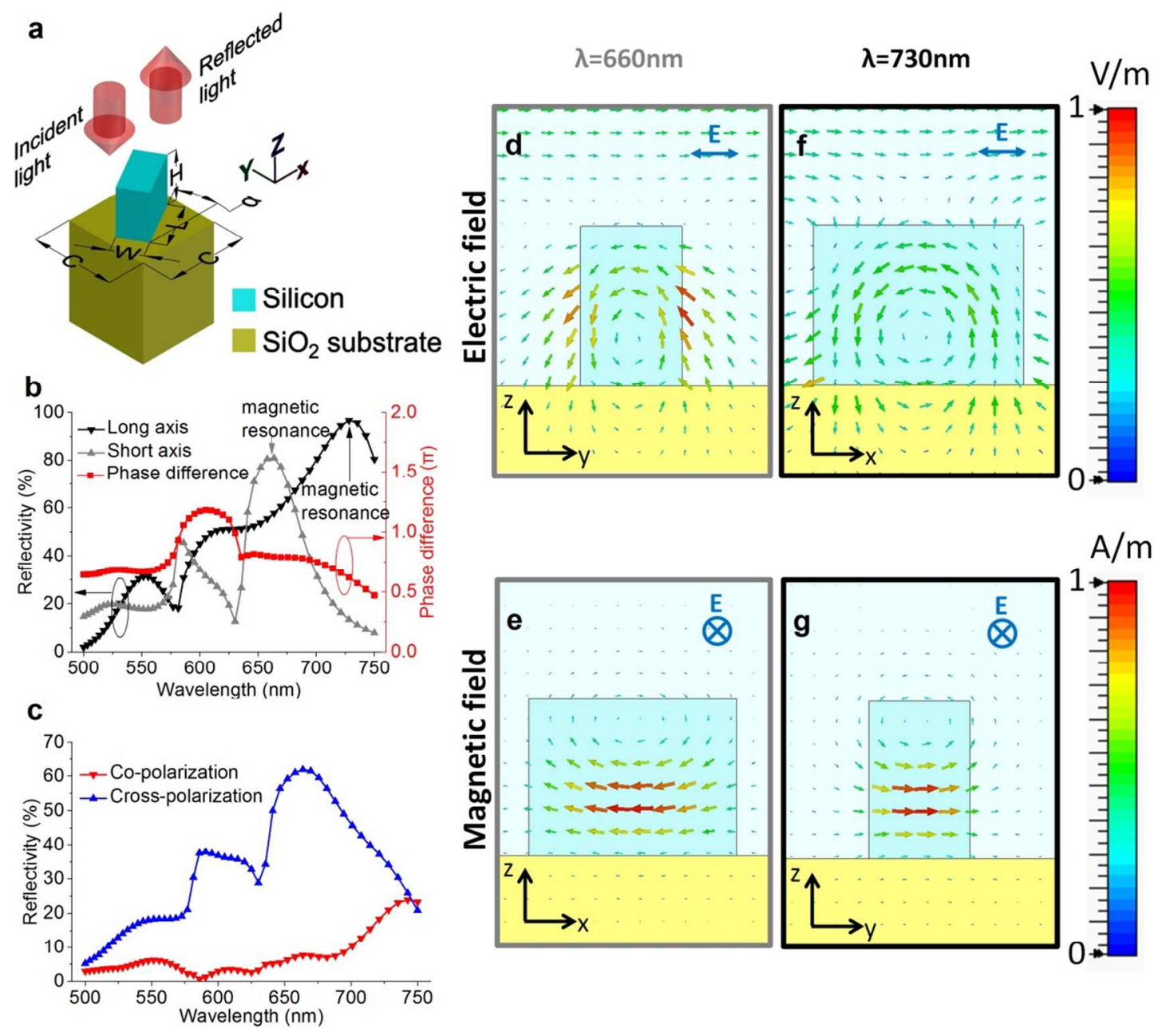

Figure 1. Illustration and simulated results of the unit cell. a, Schematic of one unit cell of the resonant GEMS.

b. Simulated reflectivity and phase difference of the nanobrick when the normal incident beam is polarized along the long axis and short axis of the nanobrick, respectively. c, Simulated reflectivity of the cross-polarized and copolarized parts under normal light incidence with circular polarization. d, e, The vortex-like normalized electric field and enhanced magnetic field distribution at the cross-section of a nanobrick unit-cell when the incident electric field is polarized along $\mathrm{y}$-axis at wavelength of $660 \mathrm{~nm}$. f, g, The vortex-like normalized electric field and enhanced magnetic field distribution at the cross-section of a nanobrick unit-cell when the incident electric field is polarized along x-axis at wavelength of $730 \mathrm{~nm}$. Polarization of incident light is indicated insets in $\mathbf{d - g}$.

Figure 1a shows the schematic of the adopted unit cell of our GEMS. Geometric phase is generated under illumination with circular polarization, and the phase delay can only be imparted 
to the reflected light with opposite polarity. Therefore, an efficient polarization conversion results in an efficient metasurface. By carefully designing the geometry size, a $\pi$ phase delay is introduced between the long and short axes and the structure then effectively acts as a half wave plate. By using the Jones calculus, a wave plate without coordinate rotation can be expressed as

$$
\mathrm{G}=\left[\begin{array}{cc}
r_{l} & 0 \\
0 & r_{s} e^{i \delta}
\end{array}\right],
$$

where $r_{l}$ and $r_{s}$ are the reflective coefficients along the long and short axes respectively and $\delta$ is the phase delay between two mutually orthogonal directions. If circularly polarized (CP) light is incident on the wave plate, the polarization conversion efficiency of the output light with ( $\left.\eta_{\text {cross }}\right)$ and without $\left(\eta_{\text {co }}\right)$ phase delay (determined by geometric phase) can be expressed as

$$
\left\{\begin{array}{l}
\eta_{\text {cross }}=\left|\frac{r_{l}-r_{s} e^{i \delta}}{2}\right|^{2} \\
\eta_{c o}=\left|\frac{r_{l}+r_{s} e^{i \delta}}{2}\right|^{2}
\end{array} .\right.
$$

In particular, only if $r_{l}=r_{s}=1$ and $\delta=\pi$, all incident CP light can be converted into the useful cross-polarized part (i.e. $\eta_{\text {cross }}=1$ and $\eta_{\mathrm{co}}=0$ ). Since varying the dimension of the nanobrick affects both the reflectivity along the long and short axes and their phase delay, we need to make a trade-off between high reflectivity and accurate phase delay of $\pi$ along two orthogonal directions. Figure $1 \mathrm{~b}$ shows the simulated reflectivity and phase difference of the nanobrick when the normally incident beam is polarized along the long axis and short axis of the nanobrick, respectively. Then we can calculate the efficiency of the reflected co-polarized part and crosspolarized part, as shown in Figure 1c. Significantly, the polarization conversion efficiency can reach as high as $60 \%$, while the unwanted co-polarized light contributing to zero-order diffraction can be suppressed to below $10 \%$. To investigate the high reflection occurring in these metasurfaces, we simulated the distribution of electric and magnetic fields at the cross-section of 
a nanobrick unit cell. As shown in Figure 1d-g, vortex-like electric fields and enhanced magnetic fields inside the nanobrick unit cell indicate the excitation of a magnetic dipole resonance along two axes. Here, the nanobrick is designed with a height $\mathrm{H}$ of $220 \mathrm{~nm}$, length $\mathrm{L}$ of $290 \mathrm{~nm}$, width $\mathrm{W}$ of $140 \mathrm{~nm}$ and cellsize $\mathrm{C}$ of $400 \mathrm{~nm}$, which leads to a height as low as $\lambda / 3$ and a low AR of 1.5 along the short axis direction. With the accompanying advantages, i.e., high efficiency, low noise, ultra-compactness and the ease for fabrication, resonant GEMS shows considerable promise for constructing high-performance phase modulators such as holograms. 
a

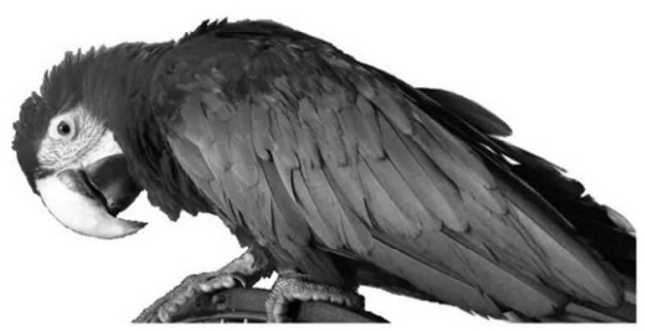

C

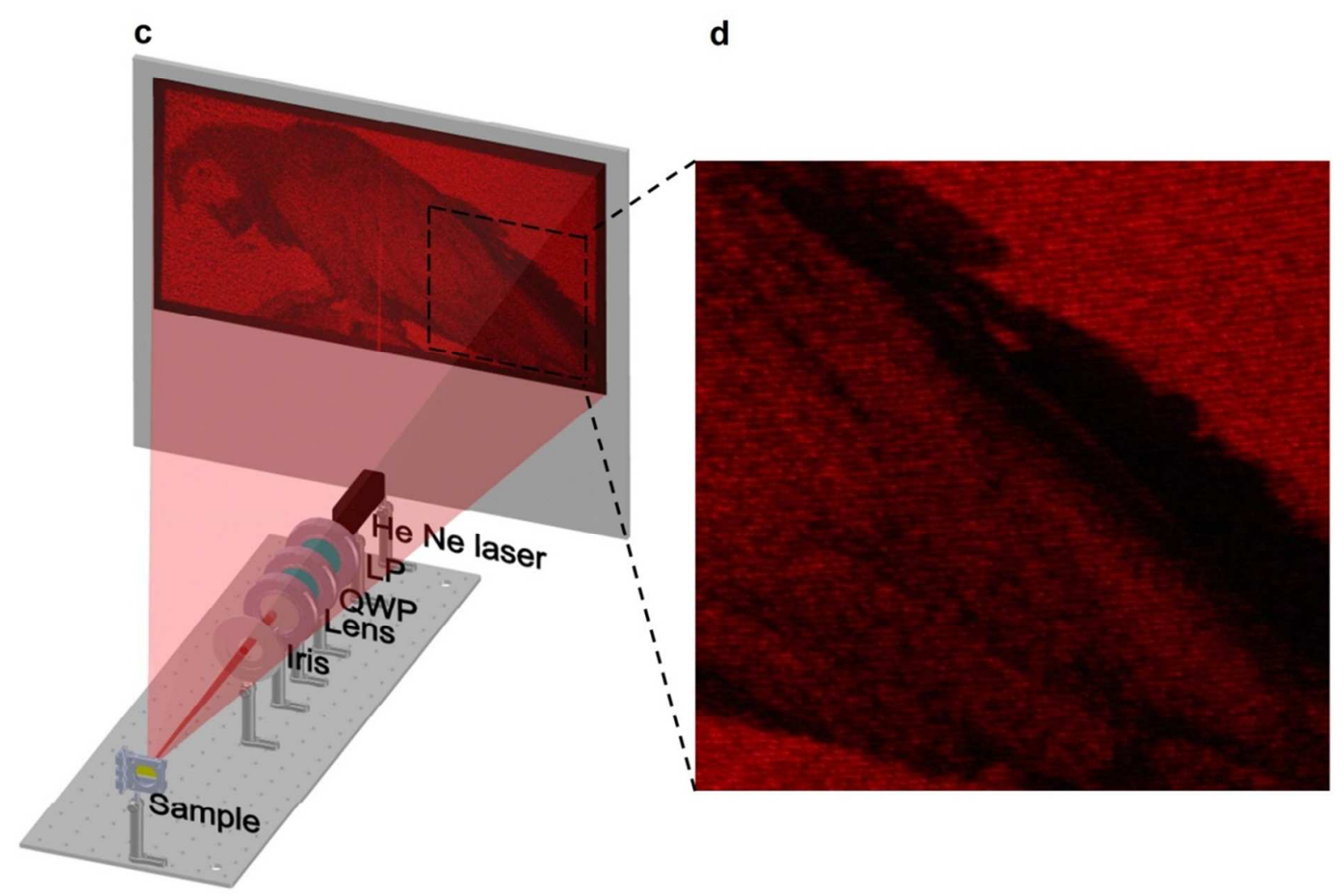

b

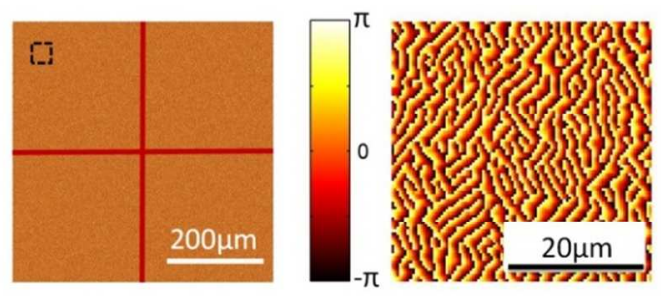

d

Figure 2. Measurement of Fourier hologram and phase distribution. a, The original object of a parrot. b, The total and partial zoom-in view $(100 \times 100$ pixels $)$ of the upper-left corner (in the dashed box $)$ phase distribution designed to generate the target holographic image in the far field. c, Illustration of the experimental setup and measured reflection-type Fourier hologram under illumination with circular polarization d, The partially zoom-in view of holographic image. The operating wavelength is $632.8 \mathrm{~nm}$ and the nanobrick arrays have the same structural parameters as illustrated in Figure 1. 
Results and Discussion. Demonstration of Fourier hologram. A Fourier hologram was designed for CP light at normal incidence, as shown in Figure 2. The reflected beam forms the holographic image (a parrot) at any plane vertical to the optical axis of incident beam in the far field. Moreover, the hologram was designed to create a wide image angle of $40^{\circ} \times 20^{\circ}$. To avoid overlap with the zero order spot, the holographic image was designed with an off-axis-angle of $10^{\circ}$. The Fourier hologram consists of $2 \times 2$ arrays, where the area of each piece is $250 \times 250$ $\mu \mathrm{m}^{2}$. The phase distribution is designed by the Gerchberg-Saxton algorithm, ${ }^{44}$ as shown in Figure 2b. The experimental (Figure 2c) holographic image, including the zoomed-in view for detail (Figure 2d), shows high fidelity of the metasurface Fourier hologram.

The efficiency measurement experimental setup is shown in Figure S1 in Supporting Information (SI). Theoretically, the meta-hologram has a polarization conversion efficiency of nearly $30 \%$ at the measured wavelength of $632.8 \mathrm{~nm}$ (as shown in Figure 1c). The measured hologram efficiency, defined as the ratio of the power of refracted holographic image and the power of incident beam, reaches $14 \%$. To further investigate the spectral response of the hologram, we measured the conversion efficiency with a super continuum light source (YSL SC-pro) in the range from 600 to $700 \mathrm{~nm}$ in steps of $10 \mathrm{~nm}$. We found that a maximum of $24 \%$ can be reached at a wavelength of $660 \mathrm{~nm}$ with an efficiency larger than $12 \%$ in the measurement range. The peak wavelength agrees well with the simulated one (as shown in Figure 1c), however the efficiency is lower than expected (60\%). Such a moderate efficiency may be attributed to several reasons. In general, the fabricated errors may cause inhomogeneous reflectivity of nanobrick arrays and greatly degrade the diffractive performance of the phase-only hologram we designed. Secondly, the diffraction efficiency is hard to be fully optimized that make all the incident beam with phase delay contributing to the reconstruction of holographic image. At last, imperfect 
shape (as shown in Figure S2 in SI) and practically larger intrinsic loss of materials will also lead to reduced efficiency. It should be noted that although the conversion efficiency in our sample might be at the lower edge of what is required for practical purposes, it arises from technical issues rather than a theoretical limit in principle. This relatively low value can be improved by more precise fabrication procedures, reducing the extending angles of holographic image and using low-loss dielectric materials (crystalline silicon or $\mathrm{TiO}_{2}$ ).

a

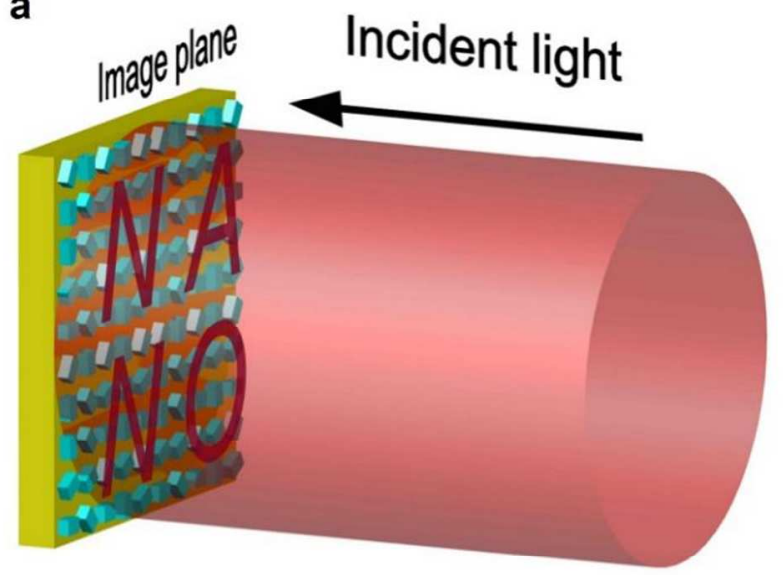

C

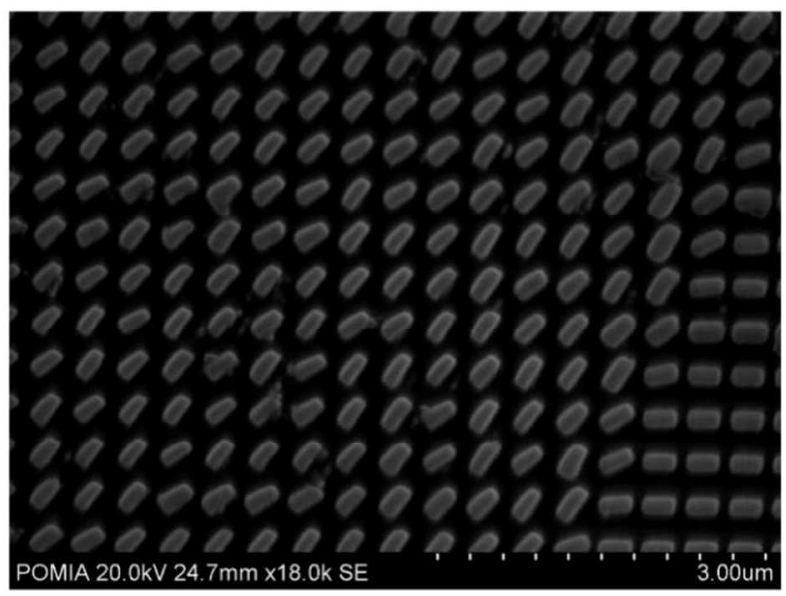

b

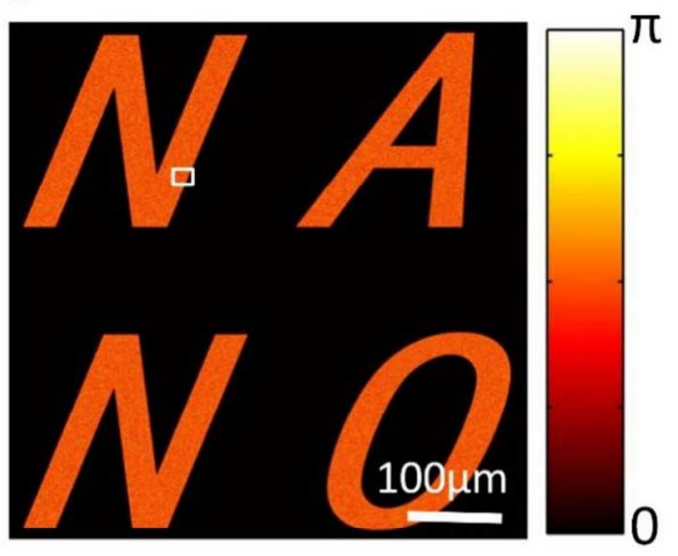

d

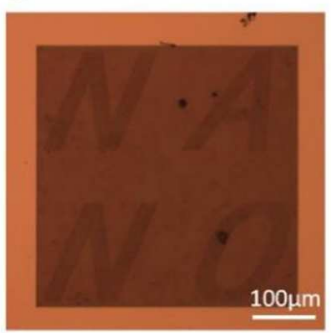

e
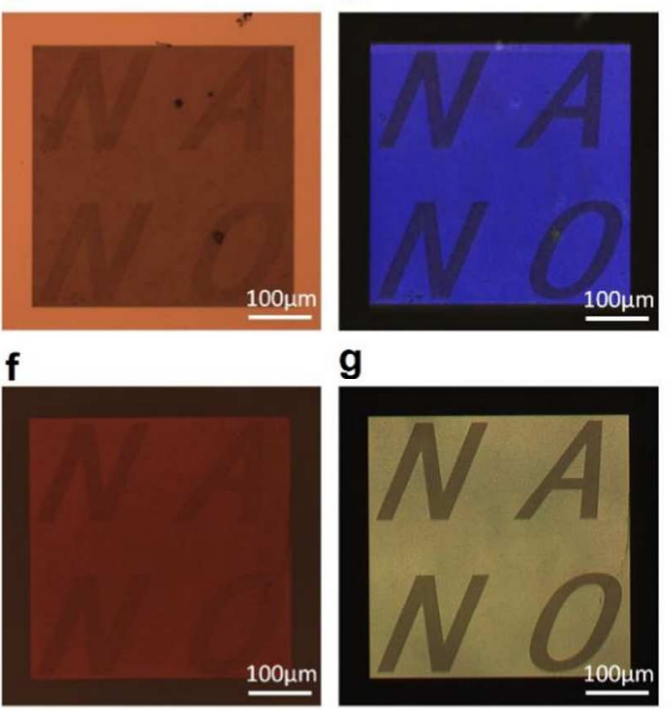

g

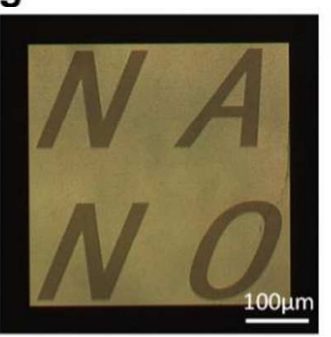

Figure 3. Working principle, phase distribution and experimental results for GEMS image hologram. a, Illustration of working principle of the dielectric GEMS image hologram. $\mathbf{b}$, The designed phase distribution to generate a binary word $N A N O$. The scale bar is $100 \mu \mathrm{m}$. The white box on the border of letter $N$ marks the area 
where SEM image is in the pattern. c, SEM image of the fabricated nanobrick arrays. d, e, Optical microscope images illuminated by a halogen lamp of microscope and a flashlight of a cellphone, respectively. The height of nanobricks is $220 \mathrm{~nm}$. f, $\mathbf{g}$, Optical microscope images illuminated by halogen lamp of the microscope and flashlight of a cellphone, respectively. The height of nanobricks is $150 \mathrm{~nm}$. Other geometry parameters are the same as those used in Figure 1.

Demonstration of image hologram with a simple pattern. Different from Fourier hologram, which produces holographic images in the far field, image holograms can render the target image floating above the sample surface, ${ }^{45}$ which can be observed by the naked eyes (if the sample is big enough) or quite conveniently through an optical microscope. Figure 3a illustrates the working principle of image hologram, where the target image is the word $N A N O$ as an example. At first, limited by computation resource, a GEMS image hologram was simulated with only $50 \times 50$ pixels at a wavelength of $658 \mathrm{~nm}$. The obtained image verifies the performance of our design (as shown in Figure S3 in SI). It is noteworthy that the grayscale difference between the character $N A N O$ and the background is mainly caused by the phase difference rather than by the magnitude difference due to the equal reflectivity of each nanobrick. Even considering the near-field coupling between neighboring nanobricks, the influences are negligible (as shown in Figure S4 in SI). More importantly, the image hologram we designed is insensitive to the polarization states of incident light. It is known that an incident beam with any polarized states can be a linear combination of two separated sub-beams with orthogonal polarization states, such as left and right circularly polarizations. By using Kirchhoff diffraction formula, we calculated the holographic image $N A N O$ produced by the phase-only element and we can get almost the same holographic images with left circularly polarized (LCP) and right circularly polarized (RCP) 
incident light (as shown in Figure S5 in SI), which agree well with the theoretical design and prove that the polarization-independent characteristics of image hologram.

Subsequently, the image hologram was fabricated by standard electron-beam lithography (EBL) with a dimension of $500 \times 500 \mu \mathrm{m}^{2}(1250 \times 1250$ pixels $)$ and the corresponding phase distribution is shown in Figure 3b. The scanning electron microscopy (SEM) image of the sample (partial view) is shown in Figure 3c. To investigate the spectral response of the metasurface hologram, we used a supercontinuum laser light (YSL SC-pro) to illuminate the sample and captured the holographic image by an optical microscope (Nikon LV150N), as shown in Figure S6 in SI. We can observe that all of the holographic images are with high fidelity and without image distortions or color blurring. The detailed mechanism of these important characteristics of image hologram has been investigated in chapter 5 of SI.

To apply to a more natural environment without a restrictive illumination condition (such as laser, polarized light, etc.), the observation of holographic image under illumination by an arbitrary light source was also performed. As shown in Figure 3d, the fabricated hologram was first illuminated by a halogen source. It is clear that the word $N A N O$ can be recognized unambiguously. The holographic image was then captured under the illumination of a flash light of a cellphone, as shown in Figure 3e. Besides a difference in color hue, the target image shows an even better contrast. The differences of the observed images may be attributed to the sample's spectral response, diffractive light from broadband source with broad illumination angles and the spectral response of charge coupled device (CCD) sensor. Because of the dissimilar spectral energy distribution and incident angle of the light sources, their diffraction behavior varies significantly. 
To further check the robustness to structure height of our design, another GEMS image hologram was fabricated with the same structure parameters and phase distribution but with a reduction in height from 220 to $150 \mathrm{~nm}$, i.e. a quarter of operation wavelength. The holographic images were also clearly observed under two types of illumination, as shown in Figures $3 \mathrm{f}-\mathrm{g}$. However, a color shift was also observed, which may be caused by the height-induced spectrum variation.

It is interesting that the GEMS image hologram is insensitive to the polarization state of incident light, which results from the virtual and real holographic images almost overlapping at the surface of hologram. Therefore, in contrast to Fresnel or Fourier holographic images, image holograms based on GEMS can work under a more relaxed illumination condition, i.e. broadband and random polarization states, from incandescent or natural light sources. These advantages make the choice of light source flexible and do not require other optical elements to observe the holographic image (coherent sources such as laser, quarter wave plate, polarizer, etc.). With these advantages, the resonant GEMS image hologram technique shows immense prospects in anti-counterfeiting, information security, display and series of fields. 


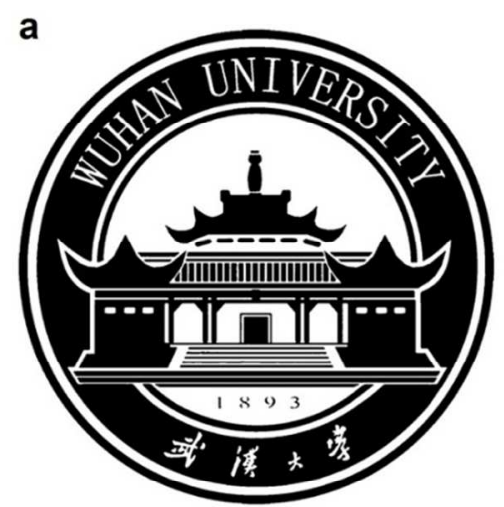

C

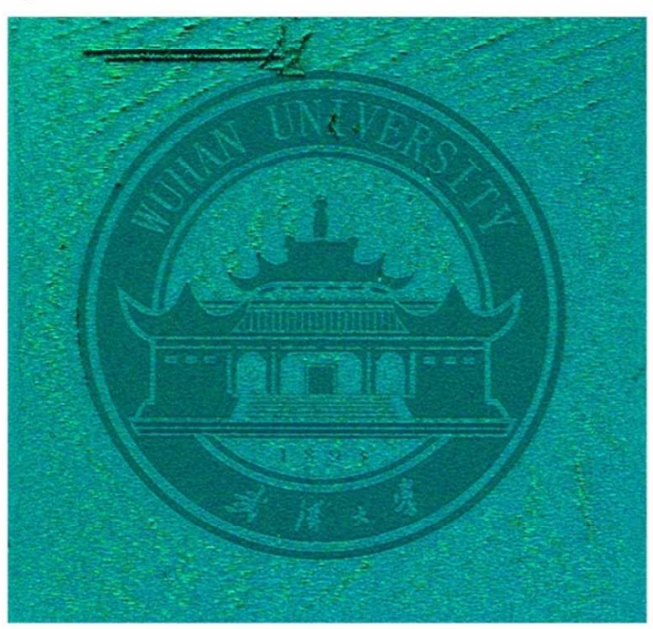

b
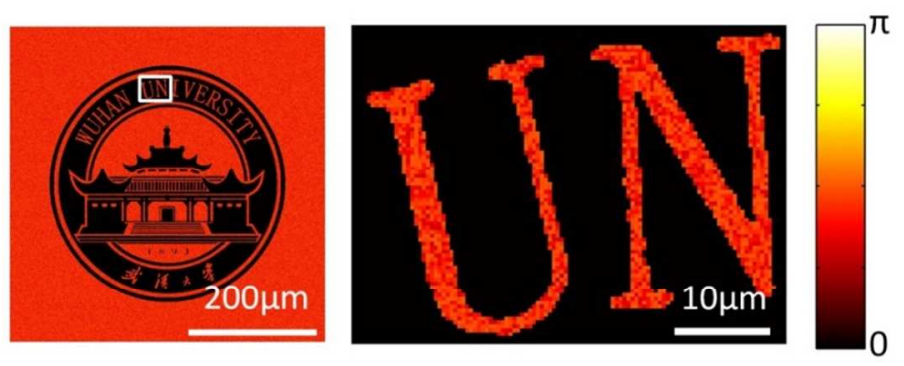

d

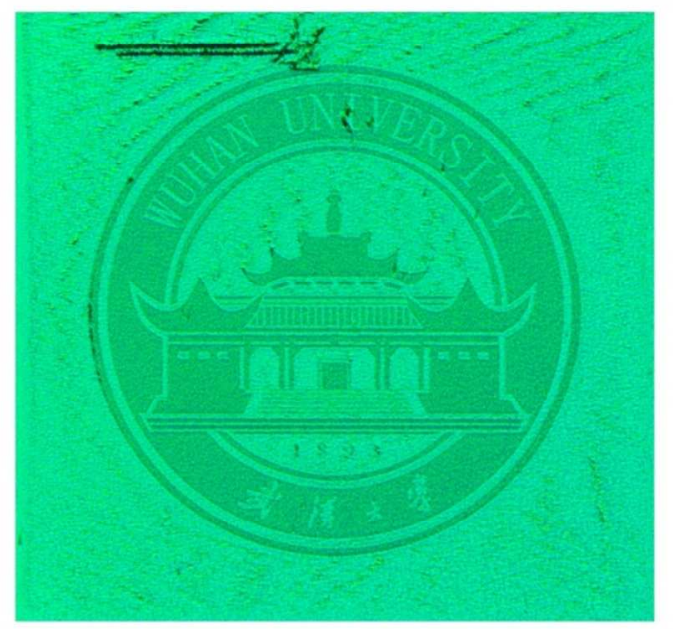

Figure 4. Experimental results for image hologram fabricated on SOI. a, The logo of Wuhan University was taken as the target image. b, The total and partial zoom-in view (in the white box) phase distribution designed to generate the logo. c, d, Optical microscope images of a resonant GEMS image holograms illuminated by natural light and a flashlight of a cellphone, respectively. The geometry parameters are the same as those used in Figure 1 except that the height is $250 \mathrm{~nm}$ here. The logo of Wuhan University is used with permission from the Wuhan University.

Demonstration of image hologram with a complicated pattern fabricated on SOI. Silicon on insulator (SOI), being widely used in integrated circuits (IC), is a promising material to make a dielectric GEMS. In our design, a SOI, consisting of a silicon dioxide $\left(\mathrm{SiO}_{2}\right)$ layer with a thickness of $2 \mu \mathrm{m}$ and a silicon cover with a thickness of $250 \mathrm{~nm}$, was employed. The logo of 
Wuhan University (Figure 4a) was taken as the target image and our designed hologram has dimensions of $500 \times 500 \mu \mathrm{m}^{2}$ (Figure $4 \mathrm{~b}$ ). The broadband response of GEMS hologram based on SOI is shown in Figure S8 in SI. Since the most promising application of the image hologram fabricated on SOI is anti-counterfeiting, the sample is illuminated by natural light and the captured image is shown in Figure 4c. We also captured the image illuminated by a flashlight of a cellphone, as shown in Figure 4d. Similar to the observations in Figure 3d-g, besides the target image, differences in color also exist when illuminated by different light sources.

Conclusions. We have presented a compact reflection-type phase-only meta-hologram using silicon nanostructure-based geometric phase at visible range. The holograms have been verified by Fourier hologram and image hologram with high fidelity. Due to the introduction of dual magnetic dipole resonances, the height of nanobricks in our GEMS can be reduced even down to $\lambda / 4$, which significantly relaxes the fabrication burden. Furthermore, the holographic image has also been demonstrated by fabricating the hologram on a commercial SOI substrate, which shows good compatibility with contemporary semiconductor industry technologies. Significantly, due to the wavelength-independent phase response, the designed hologram can be well observed under arbitrary illumination condition such as natural light, which thus may facilitate less stringent applications in the natural setting such as anti-counterfeiting. Finally, we would like to emphasize that such nanobrick-based metasurfaces can be fabricated on a large scale with much lower cost by nano-imprinting, making them promising candidates for largescale holographic technology.

Methods. Design of nanobrick unit cell. The nanobrick unit cell was designed and simulated by CST microwave studio software. In the simulation, a CP plane wave was normally incident onto a single nanobrick with periodic boundary conditions. The reflectivity of the cross-polarized and 
co-polarized parts was collected by field ports. Furthermore, we swept the geometric parameters of the nanobrick (length $\mathrm{L}$, width $\mathrm{W}$, height $\mathrm{H}$ and cellsize $\mathrm{C}$ ) to optimize the performance.

Design of Fourier hologram. In the design, a complex digital image containing a parrot with pixel number of $712 \times 351$ and 256 grayscale levels was chosen as holographic target image (Figure 2a). Because of the large angular range, the Rayleigh-Sommerfeld diffraction method was used to simulate the holographic image. ${ }^{44,46}$ The hologram was pre-compensated to avoid the geometric distortion of pattern. To avoid the formation of laser speckles in the holographic image, the concept of Dammann gratings ${ }^{47}$ was utilized for the hologram design.

Design of image hologram. Balancing the computation complexity against the diffractive performance in our design, the orientation angle $\alpha$ of each nanobrick can be expressed as

$$
\left\{\begin{array}{l}
\alpha=\frac{\pi}{4}\left(|B+R|^{2}-|B|^{2}-|R|^{2}\right) \\
\mathrm{R}=\mathrm{e}^{0.15 i \xi}
\end{array}\right.
$$

where $B$ is the brightness of the binary image ( $B$ equals 0 or 1), $R$ is the brightness of a reference beam and $\xi$ is a random number satisfies uniform distribution $U[0,1]$. There exist several alternative algorithms to optimize this phase distribution such as simulated annealing method (SA) and genetic algorithm (GA) at the cost of computing time.

Sample fabrication. The samples were fabricated on a silicon-coated $\mathrm{SiO}_{2}$ substrate with standard EBL. On a $500 \mu \mathrm{m}$ thick fused silica substrate, amorphous silicon was deposited through plasma enhanced chemical vapor deposition (PECVD). The nanobrick structures were patterned in the Copolymer (Microchem, MMA (8.5) MAA EL-8) / PMMA (Microchem, 495 PMMA A2) bilayer positive tone resists by EBL (ELIONIX ELS-7800, $80 \mathrm{kV}, 50 \mathrm{pA}$ ), and then 
$\mathrm{Cr}(40 \mathrm{~nm})$ was deposited by electron-beam evaporator (KVT KVE-ENS4004), followed by liftoff process in hot acetone. Cr was used as etch mask and Cr-free part was removed through dry etcher. After the etching process the $\mathrm{Cr}$ mask was removed by a $\mathrm{Cr}$ etchant. Finally, only silicon nanobricks remain on the substrate. In the case of SOI platform $(250 \mathrm{~nm}$ top silicon and $2 \mu \mathrm{m}$ buried oxide), the procedures are all the same except for silicon deposition process.

Experimental setup. For the Fourier hologram experiment, we used a He-Ne laser with a wavelength of $632.8 \mathrm{~nm}$. The CP laser source was incident onto the metasurface hologram, and the reflected holographic image was projected onto a white screen $300 \mathrm{~mm}$ away from the surface of the hologram. We captured the red holographic image using commercial digital cameras (PENTAX K50). For the image hologram experiment, we used the optical microscope (Nikon LV150N) to observe the holographic image directly without any other optical elements.

\section{ASSOCIATED CONTENT}

Supporting Information. Figures showing the efficiency measurement experiments of Fourier hologram, influence of fabrication defects on performance, simulation on the performance of image hologram, investigation on the working principle of image hologram and experiment on broadband response of the image hologram.

\section{AUTHOR INFORMATION}

\section{Corresponding Author}

*Email: gxzheng@whu.edu.cn, jsrho@postech.ac.kr, chengwei.qiu@nus.edu.sg

\section{Author Contributions}


Z.L., M.Q.M., G.Z., J.R. and C.W.Q. conceived and designed the experiments. Z.L. and G.Z. performed the design and simulation on the metasurfaces. I.K. fabricated the samples. Z.L., Y.W., G.Z., M.Q.M., M.S.A. and M.S. performed the measurements. Z.L., L.Z., D.L., K.T.N., B.L. and C.W.Q. analyzed the data. Z.L., L.Z., G.Z., S.Z. and C.W.Q. co-wrote the paper. All authors discussed the results and commented on the manuscript. ${ }^{\sqrt{J}}$ These authors contributed equally.

\section{ACKNOWLEDGMENT}

L.Z. acknowledges the financial support from Young Talent Recruiting Plans of Xi'an Jiaotong University and the National Natural Science Foundation of China (No. 11604256). I.K. acknowledges the Global Ph.D. fellowship from Korean government (NRF2016H1A2A1906519). M.Q.M. acknowledges the Information Technology University of the Punjab Lahore, Pakistan for financial support. G.Z. acknowledges the National Natural Science Foundation of China (Nos. 11374235, 11574240, 11774273), the Outstanding Youth Funds of Hubei Province (No. 2016CFA034), the Open Foundation of State Key Laboratory of Optical Communication Technologies and Networks, Wuhan Research Institute of Posts \& Telecommunications (No. OCTN-201605), the Fundamental Research Funds for the Central Universities (No. 2042017kf0235). J.R. acknowledges the financial supports from LGD-SNU Incubation program funded by LG Display, Green Science program funded by POSCO, and the National Research Foundation grants (NRF-2015R1C1A1A02036464, NRF2015R1A5A1037668, CAMM-2014M3A6B3063708) funded by the Ministry of Science, ICT and Future Planning (MSIP) of Korean government. C.Q. would like to acknowledge the support by the National Research Foundation, Prime Minister's Office, Singapore under its Competitive Research Program (CRP Award No. NRF-CRP15-2015-03). We also like to thank Ali Akbar for his suggestions on optical instruments. 


\section{REFERENCES}

\section{REFERENCES}

1. Chong, K. E.; Staude, I.; James, A.; Dominguez, J.; Liu, S.; Campione, S.; Subramania, G. S.;

Luk, T. S.; Decker, M.; Neshev, D. N.; Brener, I.; Kivshar, Y. S. Polarization-Independent Silicon Metadevices for Efficient Optical Wavefront Control. Nano Lett. 2015, 15, 5369-5374.

2. Li, Q.; Dong, F.; Wang, B.; Gan, F.; Chen, J.; Song, Z.; Xu, L.; Chu, W.; Xiao, Y.; Gong, Q.; Li, Y. Polarization-Independent and High-Efficiency Dielectric Metasurfaces for Visible Light. Opt. Express 2016, 24, 16309-16319.

3. Yu, N.; Genevet, P.; Kats, M. A.; Aieta, F.; Tetienne, J.; Capasso, F.; Gaburro, Z. Light Propagation with Phase Discontinuities: Generalized Laws of Reflection and Refraction. Science 2011, 334, 333-337.

4. Aieta, F.; Genevet, P.; Kats, M. A.; Yu, N.; Blanchard, R.; Gahurro, Z.; Capasso, F. Aberration-free Ultrathin Flat Lenses and Axicons at Telecom Wavelengths Based on Plasmonic Metasurfaces. Nano Lett. 2012, 12, 4932-4936.

5. Pors, A.; Nielsen, M. G.; Eriksen, R. L.; Bozhevolnyi, S. I. Broadband Focusing Flat Mirrors Based on Plasmonic Gradient Metasurfaces. Nano Lett. 2013, 13, 829-834.

6. Yang, Y.; Wang, W.; Moitra, P.; Kravchenko, I. I.; Briggs, D. P.; Valentine, J. Dielectric Meta-Reflectarray for Broadband Linear Polarization Conversion and Optical Vortex Generation. Nano Lett. 2014, 14, 1394-1399.

7. Arbabi, A.; Horie, Y.; Bagheri, M.; Faraon, A. Dielectric Metasurfaces for Complete Control of Phase and Polarization with Subwavelength Spatial Resolution and High Transmission. Nat. Nanotechnol 2015, 10, 190-937. 
8. Wang, Q.; Zhang, X.; Xu, Y.; Gu, J.; Li, Y.; Tian, Z.; Singh, R.; Zhang, S.; Han, J.; Zhang, W. Broadband Metasurface Holograms: Toward Complete Phase and Amplitude Engineering. Sci. Rep. 2016, 6, 32867.

9. Khorasaninejad, M.; Zhuit, A. Y.; Roques-Carmes, C.; Chen, W. T.; Oh, J.; Mishra, I.; Devlin, R. C.; Capasso, F. Polarization-Insensitive Metalenses at Visible Wavelengths. Nano Lett. 2016, 16, 7229-7234.

10. Yu, N.; Capasso, F. Flat Optics with Designer Metasurfaces. Nat. Mater. 2014, 13, 139-150.

11. Zheng, G.; Liu, G.; Kenney, M. G.; Li, Z.; He, P.; Li, S.; Ren, Z.; Deng, Q. Ultracompact High-Efficiency Polarising Beam Splitter Based on Silicon Nanobrick Arrays. Opt. Express 2016, 24, 6749-6757.

12. Zhou, Z.; Li, J.; Su, R.; Yao, B.; Fang, H.; Li, K.; Zhou, L.; Liu, J.; Stellinga, D.; Reardon, C. P.; Krauss, T. F.; Wang, X. Efficient Silicon Metasurfaces for Visible Light. ACS Photonics 2017, 4, 544-551.

13. Wu, C.; Arju, N.; Kelp, G.; Fan, J. A.; Dominguez, J.; Gonzales, E.; Tutuc, E.; Brener, I.; Shvets, G. Spectrally Selective Chiral Silicon Metasurfaces Based on Infrared Fano Resonances. Nat. Commun. 2014, 5, 3892.

14. West, P. R.; Stewart, J. L.; Kildishev, A. V.; Shalaev, V. M.; Shkunov, V. V.; Strohkendl, F.; Zakharenkov, Y. A.; Dodds, R. K.; Byren, R. All-Dielectric Subwavelength Metasurface Focusing Lens. Opt. Express 2014, 22, 26212-26221.

15. Deng, Z.; Zhang, S.; Wang, G. P. A Facile Grating Approach Towards Broadband, WideAngle and High-Efficiency Holographic Metasurfaces. Nanoscale 2016, 8, 1588-1594. 
16. Mehmood, M. Q.; Mei, S.; Hussain, S.; Huang, K.; Siew, S. Y.; Zhang, L.; Zhang, T.; Ling, X.; Liu, H.; Teng, J.; Danner, A.; Zhang, S.; Qiu, C. Visible-Frequency Metasurface for Structuring and Spatially Multiplexing Optical Vortices. Adv. Mater. 2016, 28, 2533-2539.

17. Mei, S.; Mehmood, M. Q.; Hussain, S.; Huang, K.; Ling, X.; Siew, S. Y.; Liu, H.; Teng, J.; Danner, A.; Qiu, C. Flat Helical Nanosieves. Adv. Funct. Mater. 2016, 26, 5255-5262.

18. Chen, X.; Huang, L.; Muehlenbernd, H.; Li, G.; Bai, B.; Tan, Q.; Jin, G.; Qiu, C.; Zhang, S.; Zentgraf, T. Dual-Polarity Plasmonic Metalens for Visible Light. Nat. Commun. 2012, 3, 1198.

19. Wang, Y.; Pu, M.; Zhang, Z.; Li, X.; Ma, X.; Zhao, Z.; Luo, X. Quasi-Continuous Metasurface for Ultra-Broadband and Polarization-Controlled Electromagnetic Beam Deflection. Sci. Rep. 2015, 5, 17733.

20. Lin, D.; Fan, P.; Hasman, E.; Brongersma, M. L. Dielectric Gradient Metasurface Optical Elements. Science 2014, 345, 298-302.

21. Khorasaninejad, M.; Crozier, K. B. Silicon Nanofin Grating as a Miniature ChiralityDistinguishing Beam-Splitter. Nat. Commun. 2014, 5, 5386.

22. Li, Z.; Zheng, G.; He, P.; Li, S.; Deng, Q.; Zhao, J.; Ai, Y. All-Silicon Nanorod-Based Dammann Gratings. Opt. Lett. 2015, 40, 4285-4288.

23. Khorasaninejad, M.; Chen, W. T.; Oh, J.; Capasso, F. Super-Dispersive Off-Axis MetaLenses for Compact High Resolution Spectroscopy. Nano Lett. 2016, 16, 3732-3737.

24. Khorasaninejad, M.; Chen, W. T.; Devlin, R. C.; Oh, J.; Zhu, A. Y.; Capasso, F. Metalenses at Visible Wavelengths: Diffraction-Limited Focusing and Subwavelength Resolution Imaging. Science 2016, 352, 1190-1194. 
25. Huang, K.; Dong, Z.; Mei, S.; Zhang, L.; Liu, Y.; Liu, H.; Zhu, H.; Teng, J.; Luk'Yanchuk, B.; Yang, J. K. W.; Qiu, C. Silicon Multi-Meta-Holograms for the Broadband Visible Light. Laser Photonics Rev. 2016, 10, 500-509.

26. Ke, Y.; Liu, Y.; He, Y.; Zhou, J.; Luo, H.; Wen, S. Realization of Spin-Dependent Splitting with Arbitrary Intensity Patterns Based on All-Dielectric Metasurfaces. Appl. Phys. Lett. 2015, 107, 0411074.

27. Ye, W.; Zeuner, F.; Li, X.; Reineke, B.; He, S.; Qiu, C.; Liu, J.; Wang, Y.; Zhang, S.; Zentgraf, T. Spin and Wavelength Multiplexed Nonlinear Metasurface Holography. Nat. Commun. 2016, 7, 11930.

28. Li, X.; Chen, L.; Li, Y.; Zhang, X.; Pu, M.; Zhao, Z.; Ma, X.; Wang, Y.; Hong, M.; Luo, X. Multicolor 3D Meta-Holography by Broadband Plasmonic Modulation. Sci. Adv. 2016, 2, e1601102.

29. Wan, W.; Gao, J.; Yang, X. Full-Color Plasmonic Metasurface Holograms. ACS Nano 2016, $10,10671-10680$.

30. Zhao, W.; Liu, B.; Jiang, H.; Song, J.; Pei, Y.; Jiang, Y. Full-Color Hologram Using Spatial Multiplexing of Dielectric Metasurface. Opt. Lett. 2016, 41, 147-150.

31. Zheng, G.; Muehlenbernd, H.; Kenney, M.; Li, G.; Zentgraf, T.; Zhang, S. Metasurface Holograms Reaching 80\% Efficiency. Nat. Nanotechnol. 2015, 10, 308-312.

32. Wen, D.; Yue, F.; Li, G.; Zheng, G.; Chan, K.; Chen, S.; Chen, M.; Li, K. F.; Wong, P. W. H.; Cheah, K. W.; Pun, E. Y. B.; Zhang, S.; Chen, X. Helicity Multiplexed Broadband Metasurface Holograms. Nat. Commun. 2015, 6, 8241.

33. Ni, X.; Kildishev, A. V.; Shalaev, V. M. Metasurface Holograms for Visible Light. Nat. Commun. 2013, 4, 2807. 
34. Huang, L.; Chen, X.; Mühlenbernd, H.; Zhang, H.; Chen, S.; Bai, B.; Tan, Q.; Jin, G.; Cheah, K.; Qiu, C.; Li, J.; Zentgraf, T.; Zhang, S. Three-Dimensional Optical Holography Using a Plasmonic Metasurface. Nat. Commun. 2013, 4, 2808.

35. Ginn, J. C.; Brener, I.; Peters, D. W.; Wendt, J. R.; Stevens, J. O.; Hines, P. F.; Basilio, L. I.; Warne, L. K.; Ihlefeld, J. F.; Clem, P. G.; Sinclair, M. B. Realizing Optical Magnetism from Dielectric Metamaterials. Phys. Rev. Lett. 2012, 108, 0974029.

36. Liu, S.; Sinclair, M. B.; Mahony, T. S.; Jun, Y. C.; Campione, S.; Ginn, J.; Bender, D. A.; Wendt, J. R.; Ihlefeld, J. F.; Clem, P. G.; Wright, J. B.; Brener, I. Optical Magnetic Mirrors without Metals. Optica 2014, 1, 250-256.

37. Zhao, Q.; Zhou, J.; Zhang, F.; Lippens, D. Mie Resonance-Based Dielectric Metamaterials. Mater. Today 2009, 12, 60-69.

38. Moitra, P.; Slovick, B. A.; Li, W.; Kraychencko, I. I.; Briggs, D. P.; Krishnamurthy, S.; Valentine, J. Large-Scale All-Dielectric Metamaterial Perfect Reflectors. ACS Photonics 2015, 2, 692-698.

39. Shalaev, M. I.; Sun, J.; Tsukernik, A.; Pandey, A.; Nikolskiy, K.; Litchinitser, N. M. HighEfficiency All-Dielectric Metasurfaces for Ultra-Compact Beam Manipulation in Transmission Mode. Nano Lett. 2015, 15, 6261-6266.

40. Kuznetsov, A. I.; Miroshnichenko, A. E.; Fu, Y. H.; Zhang, J.; Luk Yanchuk, B. Magnetic Light. Sci. Rep. 2012, 2, 492.

41. Kuznetsov, A. I.; Miroshnichenko, A. E.; Brongersma, M. L.; Kivshar, Y. S.; Luk Yanchuk, B. Optically Resonant Dielectric Nanostructures. Science 2016, 354, 2472. 
42. Yu, Y. F.; Zhu, A. Y.; Paniagua-Dom, R.; Fu, Y. H.; Luk Yanchuk, B.; Kuznetsov, A. I. High-Transmission Dielectric Metasurface with $2 \pi$ Phase Control at Visible Wavelengths. Laser Photonics Rev. 2015, 9, 412-418.

43. Decker, M.; Staude, I.; Falkner, M.; Dominguez J.; Neshev, D. N.; Brener, I.; Pertsch, T.; Kivshar, Y. S. High-Efficiency Dielectric Huygens' Surfaces. Adv. Opt. Mater. 2015, 3, 813820.

44. Gerchberg, R. W.; Saxton, W. O. A Practical Algorithm for the Determination of Phase from Image and Diffraction Plane Pictures. Optik 1972, 35, 237.

45. Rebane, A.; Drobizhev, M.; Sigel, C. Single Femtosecond Exposure Recording of an Image Hologram by Spectral Hole Burning in an Unstable Tautomer of a Phthalocyanine Derivative. Opt. Lett. 2000, 25, 1633-1635.

46. Shen, F.; Wang, A. Fast-Fourier-Transform Based Numerical Integration Method for the Rayleigh-Sommerfeld Diffraction Formula. Appl. Opt. 2006, 45, 1102-1110.

47. Dammann, H.; Görtler, K. High-Efficiency In-Line Multiple Imaging by Means of Multiple Phase Holograms. Opt. Commun. 1971, 3, 312-315.

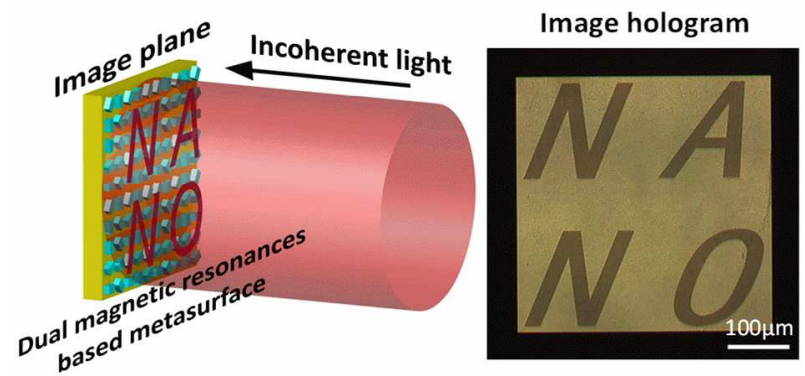

For Table of Contents Only 